\title{
Jet lag prevented in mutant mice
}

Mice lacking vasopressin V1a and V1b receptors avoid jet lag, report researchers from Japan.

"Jet lag symptoms arise from temporal misalignment of the endogenous circadian clock with external time, but the specific cellular and molecular mechanisms of jet lag are unknown," says senior researcher Hitoshi Okamura of Kyoto University. "We think that the suprachiasmatic nucleus (SCN), where the master clock is located, might hold the key."

The researchers generated several mutant mice that lacked genes which are normally highly expressed in the SCN. The investigators then exposed these mice to experimental jet lag by advancing their light-dark cycles by $8 \mathrm{~h}$. Among the mutant mice screened were those that lacked vasopressin V1a and V1b receptors; these mice immediately re-entrained to the new light-dark cycle, in contrast to wild-type mice, which took 8-10 days to re-entrain. Importantly, these mutant mice had a functional oscillating clock in normal light-dark conditions and normal clock gene expression.

In vitro experiments further suggested that vasopressin signalling in the SCN contributes to interneuronal communication that confers resistance against external perturbation of the internal clock. In addition, the investigators showed that pharmacological blockade of vasopressin V1a and V1b receptors in the SCN of wild-type mice improves recovery from jet lag.

The findings hint at a potential target for the development of an anti-jet-lag therapeutic in humans, which might also mitigate the adverse metabolic consequences of shift work.

\section{Carol Wilson}

Original article Yamaguchi,Y. et al. Mice genetically deficient in vasopressin $\mathrm{V} 1 \mathrm{a}$ and $\mathrm{V} 1 \mathrm{~b}$ receptors are resistant to jet lag. Science 342, 85-90 (2013) 